\title{
Challenges and Applications of High Spatial and Energy Resolution EELS for Mapping Functional Chemistry in Beam-Sensitive Materials at Low Acceleration Voltages
}

Quentin Ramasse ${ }^{1,2^{*}}$, Demie Kepaptsoglou ${ }^{1,3}$, Christian Vollmer ${ }^{4}$, Fredrik Hage ${ }^{1}$ Patricia Abellan $^{1}$ and Jan Leitner ${ }^{5}$

1. SuperSTEM Laboratory, SciTech Daresbury Campus, Daresbury, United Kingdom

2. School of Chemical and Process Engineering and School of Physics, University of Leeds, Leeds, United Kingdom

3. Department of Physics and JEOL Nanocentre, University of York, York, United Kingdom

4. Institut für Mineralogie, Westfälische Wilhelms-Universität, Münster, Germany

5. Max Planck Institute for Chemistry, Mainz, Germany

* Corresponding author: qmramasse@superstem.org

Recent instrumentation advances have pushed the spatial resolution of mid- to low-acceleration voltage $(<60 \mathrm{keV})$ scanning transmission electron microscopes (STEMs) below $0.1 \mathrm{~nm}$, while providing energy resolution for electron energy loss spectroscopy (EELS) of under $10 \mathrm{meV}$. For materials such as graphene for which knock-on is the dominant beam damage mechanism, these 'gentle' conditions have truly realized the promise of placing a 'synchrotron in a microscope' [1]. High spatial and energy resolution EELS at acceleration voltages below the knock-on threshold for carbon can be used to unambiguously confirm the $p$ and $n$ character induced in graphene through the inclusion of single atom $\mathrm{B}$ or $\mathrm{N}$ substitutional dopants and map the localized changes in plasmonic and optical response at the atomic scale [2]. The foreign atoms do however modify the energetics of the system, and can locally weaken bonds enough that some ejections are possible, even at $60 \mathrm{keV}$. Understanding the energy transfer mechanisms from the electron beam to the sample and harnessing sub-impact-threshold events by adjusting the operating conditions can even lead to promising new methods for the direct assembly of nanostructures and atomically-precise manipulation with a STEM probe [3].

However, such an approach is not always possible and electron beam damage can be severe enough to make high resolution EELS, an intrinsically dose-hungry technique, quite a challenge. This is the case in particular of many organic systems: energy resolution, beam current and electron optics must then be adjusted and traded off each other within the greatly increased range afforded by modern microscopes. This is demonstrated in a study aiming to map the local functional chemistry and bonding nature in amorphous organic grains in meteoritic samples, namely carbonaceous chondrites. These materials are of particular importance as they are candidates in the study of ancient organic compounds as precursors for the prebiotic history of the early Earth. In order to elucidate the functional chemistry of these materials and understand the complex processes of formation and consecutive alterations they might have undergone in the molecular cloud where they were formed, monochromated STEM EELS measurements at resolution below $100 \mathrm{meV}$, under low acceleration voltage and controlled beam current, are essential. These conditions, below the $\mathrm{C}$ knock on damage threshold but also crucially at electron doses still tolerable for the organic grains, allow for a detailed observation of the pristine chemistry of the organics; the carbon and nitrogen near edge fine structures (ELNES) reveal the presence of different functional groups, namely aliphatic and carboxyl bonds, on a submicron scale, which can be attributed to early cometary and meteoritic organic reservoirs [4]. The observation of the vibrational bands associated with these specific functional chemistries from the very same sample location used for 
analytical electron microscopy confirms unambiguously that the functional groups are still intact and present, in spite of the challenging sample preparation procedure. This makes possible to characterize the optical properties of nanomaterials on a scale not accessible before [5]: organic matter in meteorites has been characterized for a long time by Raman spectroscopy techniques on a $\mu \mathrm{m}$ scale but never in correlation with functional group mapping on the nm scale. [6]

\section{References:}

[1] Q Ramasse, Ultramicroscopy 180 (2017), p. 41.

[2] F. Hage et al., Submitted (2019).

[3] T Susi et al., 2D Materials 4, 042004 (2017)

[4] C Vollmer et al., Meteoritics \& Planetary Science 53(S1) (2018), p. 6109.

[5] C Vollmer et al., Submitted (2019).

[6] SuperSTEM is the UK National Research Facility for Advanced Electron Microscopy, supported by the Engineering and Physical Sciences Research Council (EPSRC).
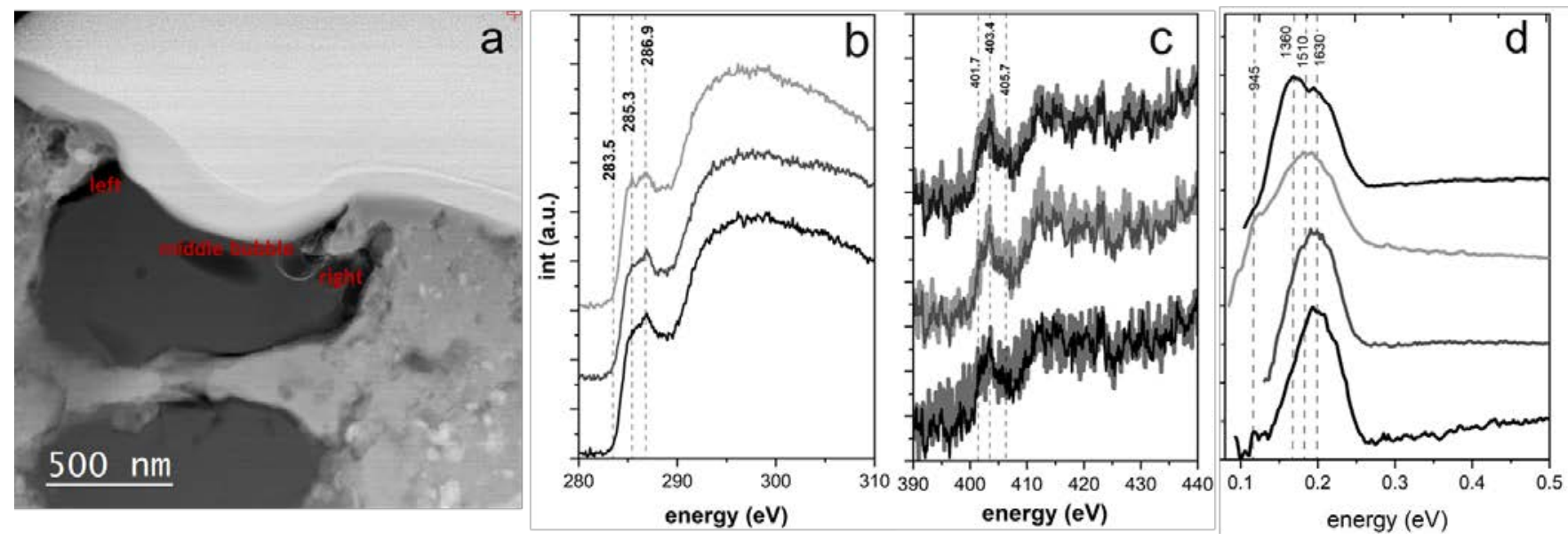

Figure 1. a) HAADF STEM image of an amorphous organic globular grain in the carbonaceous chondrite Renazzo, with spatially resolved variations across the grain (on the left, middle and right side, as indicated) of the EELS near-edge fine structure of the b) $\mathrm{C} K$ edge, c) $\mathrm{N} K$ edge and d) vibrational response [5]. The energy resolution for core loss mapping was set at $100 \mathrm{meV}$, while it was increased to $\sim 20 \mathrm{meV}$ for vibrational mapping. Beam current and probe size were controlled to mitigate beam damage to the organic matter. 